\title{
Upgrades of beam diagnostics with application to emittance-exchange experiments
}

\author{
A. H. Lumpkin, A. S. Johnson, J. Ruan, J. Santucci, Y.-E Sun, R. Thurman-Keup, and H. Edwards \\ Fermilab, Batavia, Illinois 60510, USA \\ (Received 15 January 2011; published 10 June 2011)
}

\begin{abstract}
The possibility of using electron-beam phase-space manipulations to support a free-electron laser accelerator design optimization has motivated our research. An ongoing program demonstrating the exchange of transverse horizontal and longitudinal emittances at the Fermilab A0 photoinjector has benefited recently from the upgrade of several of the key diagnostics stations. Accurate measurements of these properties upstream and downstream of the exchanger beam line are needed. Improvements in the screen resolution term and reduced impact of the optical system's depth of focus by using YAG:Ce single crystals normal to the beam direction will be described. The requirement to measure small energy spreads $(<10 \mathrm{keV})$ in the spectrometer and the exchange process which resulted in bunch lengths less than $800 \mathrm{fs}$ led to other diagnostics performance adjustments and upgrades as well. Application of these diagnostics in demonstrating the exchange of transverse $(x)$ and longitudinal phase spaces is also reported.
\end{abstract}

DOI: 10.1103/PhysRevSTAB.14.060704

PACS numbers: 41.60.Ap, 41.60.Cr

\section{INTRODUCTION}

It is recognized that beam manipulations such as a flat beam transformation followed by an emittance exchange (EEX) could support a high gain free-electron laser (FEL) push to shorter wavelengths [1,2]. An ongoing program demonstrating the exchange of transverse horizontal and longitudinal emittances at the Fermilab A0 photoinjector (A0PI) addresses the latter of these beam manipulations. Recent upgrades to key optical diagnostics stations have improved our capabilities to measure EEX. The experiments rely on accurate measurements of the emittance properties upstream and downstream of the exchanger beam line. At gamma 30, the nominal transverse beam sizes of $1 \mathrm{~mm}(\sigma)$ were not an imaging challenge. However, resolution limits are approached with the use of an array of 50- $\mu \mathrm{m}$ wide slits that sample the transverse phase spaces in order to measure divergences of less than $100 \mu \mathrm{rad}$. Such low divergences are evidenced by typically 20 times smaller slit images than the initial beam size. The 1-mm spacing of the slits also resulted in images with positions distributed over several $\mathrm{mm}$ which involved the depth-of-focus limits of the initial optics system. Improvements in the screen resolution term and reduction of the system depth-of-focus impact by using YAG:Ce single crystals normal to the beam direction will be described.

On the longitudinal side, the requirement to measure small energy spreads $(<10 \mathrm{keV})$ in the spectrometer and bunch lengths less than $800 \mathrm{fs}$ impacted the corresponding diagnostics performance specifications as well. Upgrades

Published by the American Physical Society under the terms of the Creative Commons Attribution 3.0 License. Further distribution of this work must maintain attribution to the author(s) and the published article's title, journal citation, and DOI. to the electron spectrometer focal plane screens and to the Hamamatsu C5680 streak camera (and the addition of a Martin-Puplett interferometer) addressed the energy spreads and short bunch lengths, respectively, generated by the exchange process. Following a discussion of the critical aspects of beam-size imaging diagnostics and the upgrades in Secs. II and III, examples of the EEX results obtained with the upgraded diagnostics will be presented in Secs. IV and V.

\section{EXPERIMENTAL ASPECTS}

The tests were performed at the Fermilab A0 photoinjector facility which includes an L-band photocathode (PC) rf gun and a nine-cell superconducting radiofrequency accelerating structure which combine to generate up to $16-\mathrm{MeV}$ electron beams $[3,4]$. The $\mathrm{Nd}$ glass drive laser operates at $81.25 \mathrm{MHz}$ with the micropulse structure counted down to $1 \mathrm{MHz}$. The frequency-quadrupled component at $263 \mathrm{~nm}$ is used to irradiate the $\mathrm{Cs}_{2} \mathrm{Te}$ PC. Because of the low electron-beam energies (14-16 MeV), one radiation converter type, and/or slit sampling, we typically summed over micropulses in such cases to obtain adequate signal strength in the CCD camera. The typical beam parameters for the initial conditions used in these experiments are listed in Table I. The 250-pC micropulse charge was chosen as a compromise between possible space-charge effects and diagnostic signal strength.

Fundamental to the observations of EEX were the careful beam transport and characterization of the transverse and longitudinal emittances before and after the process. The A0PI beam lines provided these capabilities through a set of rf beam position monitors and optical diagnostics stations as shown in Fig. 1. These imaging stations (crosses denoted with an "X\#") use optical transition radiation 
TABLE I. Initial electron-beam parameters of the AOPI facility. The UV drive laser pulse was $2.5 \pm 0.2 \mathrm{ps}(\sigma)$.

\begin{tabular}{lcc}
\hline \hline Parameter & Value & Units \\
\hline Energy & $14.3 \pm 0.1$ & $\mathrm{MeV}$ \\
Micropulse charge & $250 \pm 20$ & $\mathrm{pC}$ \\
Beam size $(\sigma)$ & $1-2$ & $\mathrm{~mm}$ \\
Divergence $(\sigma)$ & $50-100$ & $\mu \mathrm{rad}$ \\
Bunch length $(\sigma)$ & $2.9 \pm 0.3$ & $\mathrm{ps}$ \\
Emittance (normalized) & $2.6 \pm 0.3$ & $\mathrm{~mm} \mathrm{mrad}$ \\
\hline \hline
\end{tabular}

(OTR) $[5,6]$ and/or scintillator converter screens. Since the initial report of observation of EEX [7], we have upgraded all of the divergence and spectrometer stations to dramatically reduce the magnitude of needed corrections to the simple image sizes that relate to beam size, divergence, or energy spread depending on the diagnostics configuration. All data were obtained with a micropulse charge of $250 \mathrm{pC}$, but in some cases 10-50 micropulses were used in a single macropulse integrated by the camera CCD chip. In all cases the photocathode-dark-current signal was subtracted. The dark-current background subtraction is directly implemented by using the laser shutter to provide a dark-current-only image for subtraction from the transverse distributions. The dark-current spatial distribution is generally different and displaced somewhat from that of the photoelectrons because they are generated at different gun phases (and hence energies). In the longitudinal side, this originating phase difference of 20 to 25 degrees off crest in the rf gun separated the photoelectron streak image in time from the spread out dark-current image cleanly, and the resulting energy differences physically separated the sources also in the electron spectrometer.

The former YAG:Ce 50- $\mu \mathrm{m}$ thick powder screens on an $\mathrm{Al}$ substrate oriented at 45 degrees to the beam direction [7,8] (with updated spatial resolution term in Sec. III) have been now replaced by $100-\mu \mathrm{m}$ thick YAG:Ce single crystals oriented normal to the beam direction followed by a $45^{\circ}$ mirror to direct the radiation to the optical system. This configuration reduces the screen resolution term to less than $10 \mu \mathrm{m}$ rms based on evaluation of previous reports $[9,10]$ and also eliminates the depth-of-focus issue of the $45^{\circ}$ scintillator for multiple slit images spread across several $\mathrm{mm}$ of the field of view.

Energizing a downstream horizontal bending dipole sends the beam into a final beam dump and provides a spectrometer capability when combined with imaging of the beam size at the XS3 dispersive point in the focal plane. The depth-of-focus effect was also addressed for beam image location in the direction perpendicular to the energy-dispersive direction in the spectrometers by the same configuration change to a YAG:Ce crystal and mirror. The dispersion in $x$ is $324 \mathrm{~mm}$, and the CCD calibration factor is $47.5 \mu \mathrm{m}$ per pixel. This means a $0.1 \%$ energy spread would correspond to an $\sim 324-\mu \mathrm{m}$ beam size after corrections are done.

The initial emittance sampling station was chosen at X3, and an optical transport system using a 50-mm diameter field lens and a flat mirror brought the light to the FireWire digital CCD camera with a $25-\mathrm{mm}$ focal length C-mount lens. In addition, a larger drift length for the initial divergence measurements was attained by using the X05 and X06 stations (drifts of 0.79 and $1.29 \mathrm{~m}$, respectively) instead of X04 and X05 to view the slit images whose sizes were now dominated by the divergence-term contribution. The system edge-resolution-function terms were evaluated for each camera with standard line-pair patterns and were typically about $30-40 \mu \mathrm{m}$, and the rms contribution of the finite slit width size was evaluated as $14 \mu \mathrm{m}$. These were subtracted in quadrature from the observed slitimage profile sizes. So the projected beam sizes were recorded at X3 using OTR, and then vertical and horizontal slit assemblies were inserted in sequence at this position per the slits-emittance-measuring technique [11]. These slit assemblies were 3-mm thick tungsten plates separated with spacers to form 50- $\mu \mathrm{m}$ wide slits. The depth of metal was chosen to provide strong opacity to the $14.3-\mathrm{MeV}$ beam. A MATLAB-based graphical user interface program

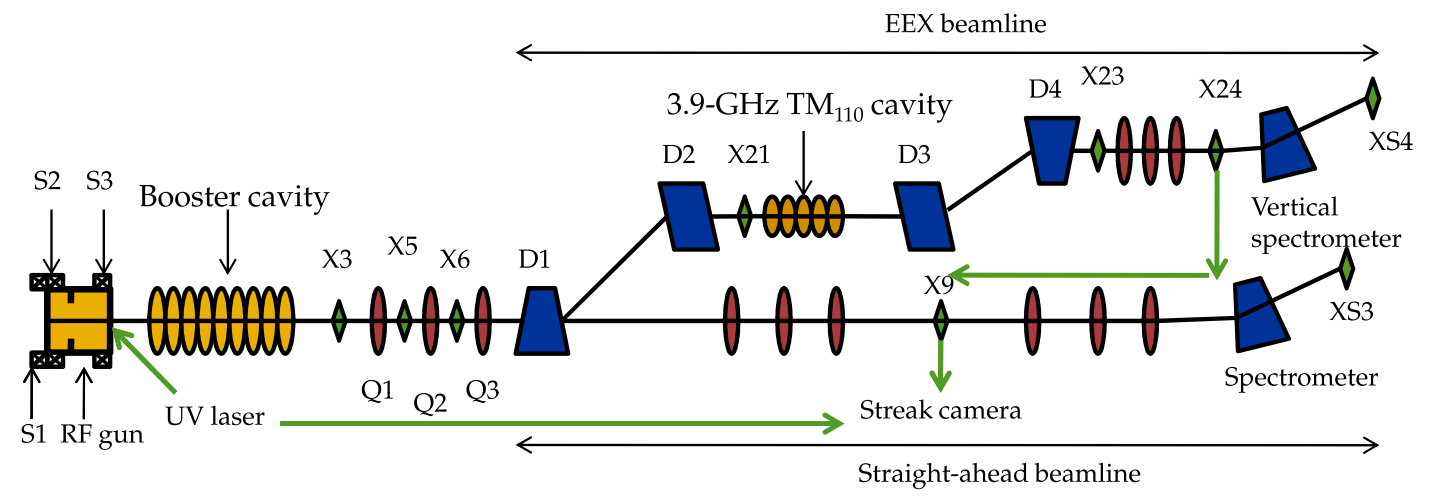

FIG. 1. A schematic of the A0 photoinjector test area showing the PC $\mathrm{rf}$ gun, nine-cell booster cavity, transverse emittance stations, the OTR stations, the streak camera, and the EEX beam line with the two doglegs, five-cell deflecting mode cavity, transverse emittance stations, and spectrometer. 
calculated the emittances and Courant-Snyder (C-S) parameters $(\alpha, \beta, \gamma)$ based on the X3-X5 and X3-X6 image pairs [12]. This program also applied the specific calibration factors and resolution terms for each camera, provided the fit parameters for the projected profiles of beam size and divergences, and calculated the statistical uncertainties. The uncertainties also include the uncertainty on the optical magnification, the resolution, and slit widths. The weighted average of the sampled divergences was used, with the weighting being the inverse uncertainty squared [12]. Alternatively, the 4-dipoles (D1-D4) of the emittanceexchange (EEX) line could be powered and emittance measurements done at an OTR Cross X23 after the fourth dipole with a drift of $0.56 \mathrm{~m}$ to Cross X24. The latter has both OTR and YAG:Ce crystal screen options. The OTR converter was an Al-coated optics mirror with a $1.0-\mathrm{mm}$ thick glass substrate, and was mounted with its surface at 45 degrees to the beam direction on a stepper assembly. The X24 assembly provided vertical positioning with an option for a YAG:Ce crystal scintillator position. The photoelectron charge of a single bunch was monitored by an upstream current monitor.

\section{SYSTEM RESOLUTION EFFECTS}

There are several contributions to the observed beam image size. When they are uncorrelated, the terms can be added in quadrature as described by Lyons [13]. The terms that have been considered are actual beam size (act), camera resolution (cam), YAG:Ce screen effects (YAG), and the finite slit width (slit) as shown here:

$$
\mathrm{obs}^{2}=\mathrm{act}^{2}+\mathrm{YAG}^{2}+\mathrm{cam}^{2}+\mathrm{slit}^{2},
$$

and after solving for the actual beam size we have

$$
\text { act }=\left[\mathrm{obs}^{2}-\mathrm{YAG}^{2}-\mathrm{cam}^{2}-\mathrm{slit}^{2}\right]^{1 / 2} .
$$

In the spectrometer, the slit width is replaced by the beam size without dispersion, $\beta_{x} \varepsilon_{x} / \gamma$ (where $\gamma$ is the Lorentz factor and $\varepsilon_{x}$ is the normalized $x$ emittance), and the actual beam size is now the dispersive term-energyspread product $\left(\eta_{x} \sigma_{E}\right)$, and this gives

$$
\mathrm{obs}^{2}=\mathrm{act}^{2}+\mathrm{YAG}^{2}+\mathrm{cam}^{2}+\beta_{x} \varepsilon_{x} / \gamma,
$$

and after solving for the actual beam size we have

$$
\text { act }=\left[\mathrm{obs}^{2}-\mathrm{YAG}^{2}-\mathrm{cam}^{2}-\beta_{x} \varepsilon_{x} / \gamma\right]^{1 / 2}=\eta_{x} \sigma_{E} .
$$

As mentioned in Sec. II, we subtracted the cameraresolution term and the finite slit width as appropriate for each station. The screen resolution term was identified as needing more consideration which we describe in Sec. III A below. Ultimately the single crystal solution gave a YAG term which was $\sim 6$ times smaller than the YAG powder term, 3 times smaller than the cameraresolution terms, and $40 \%$ smaller than the slit term so this became negligible in the quadrature sum and within the statistical error spread. Also, when we used a powder screen at 45 degrees, the depth-of-focus aspect provided a practical complication of a " $z$-dependent" cameraresolution term as discussed with its solution in Sec. III B.

\section{A. Scintillator screen resolution term}

One of the main characteristics of powder scintillator screens in the past has been a limiting resolution term that was screen-thickness dependent such as found in $\mathrm{Al}_{2} \mathrm{O}_{3}: \mathrm{Cr}$ samples $[14,15]$. Another aspect involves the grain size of the scintillator particles since this should also contribute to the limiting resolution term. More recent versions of thin YAG:Ce powder screens utilized at our linac involved reported grain sizes at the 5- $\mu \mathrm{m}$ level [16]. However, we report our comparisons with OTR screen results that indicate that the $50-\mu \mathrm{m}$ thick layer deposited on a $1-\mathrm{mm}$ substrate and oriented at 45 degrees to the beam direction has a 1-sigma resolution term much larger than the grainsize value. Our investigations were limited by the nominal beam sizes available at this $16-\mathrm{MeV}$ photoinjector so slit images as well as focused beam stripe images were used to provide a source term in one transverse dimension small enough to detect these effects. The studies were also complicated by the roughly 100 times stronger signal of the scintillator compared to the OTR screen for a given charge, and the effects of the rf amplitude and phase flatness over a pulse train. Our initial comparison of the powder screen and OTR in the same geometry is shown in Table II. For comparison purposes, we also show the OTR polarization data in the table. First, it is noted that

TABLE II. A comparison of beam image sizes (narrow dimension of vertical stripes) using YAG:Ce powder and OTR screens (both oriented at 45 degrees to the beam direction) at station $\mathrm{X} 5$. The linear polarizer effect with OTR is also shown. The YAG:Ce powder data used only one bunch as denoted by $1-\mathrm{Y}$ in column 4 . Averages were based on Gaussian fits to the projected profiles of ten images, and the variances of values of the sigma fits were determined.

\begin{tabular}{lclc}
\hline \hline X5 linear polarization & No. bunches & Fit sigma (pixels) & Size $(\mu \mathrm{m})$ \\
\hline None & 10 & $5.49 \pm 0.05$ & 124.5 \\
Vertical & 10 & $4.47 \pm 0.09$ & 101 \\
None & $1-Y$ & $5.67 \pm 0.09$ & 128.7 \\
Vertical & $1-Y$ & $5.71 \pm 0.04$ & 129.6 \\
\hline \hline
\end{tabular}


there is evidence for an OTR polarization effect where one uses the perpendicular polarization component to assess a given beam-size dimension $[17,18]$ in rows 1,2 , and, second, no polarization effect observed as expected in the YAG:Ce based beam sizes at rows 3,4. Only one bunch was used with the brighter scintillator, so we would be reducing any macropulse effects in the beam in this case. The vertically polarized OTR band images in row 2 have a projected profile size average of $101 \mu \mathrm{m}$ compared to the total OTR image size average of $124 \mu \mathrm{m}$ and the YAG:Ce image size average of $129 \mu \mathrm{m}$. These results are consistent with our previous hypothesis that the YAG:Ce powder screen has a limiting resolution term to be addressed [8]. If we accept the OTR polarized data as the reference, then the implied YAG:Ce X5 screen term in quadrature would be $80 \pm 10 \mu \mathrm{m}$ although the mean value would be $40 \mu \mathrm{m}$ if the total OTR image size were used as reference. In a second series of tests the powder screen images were compared to those of a YAG:Ce single crystal with surface normal to the beam direction, and a resulting estimate of 50-60 $\mu \mathrm{m}$ for the powder term was obtained. We averaged these three results to obtain our resolution value of $60 \pm 20 \mu \mathrm{m}$ for the present screens obtained from DESY [16].

As further context, we collect the results of several reports in the literature $[14,15,19]$ where the powder screen beam image sizes were compared directly to either those of OTR or a YAG:Ce single crystal. Again we evaluated the

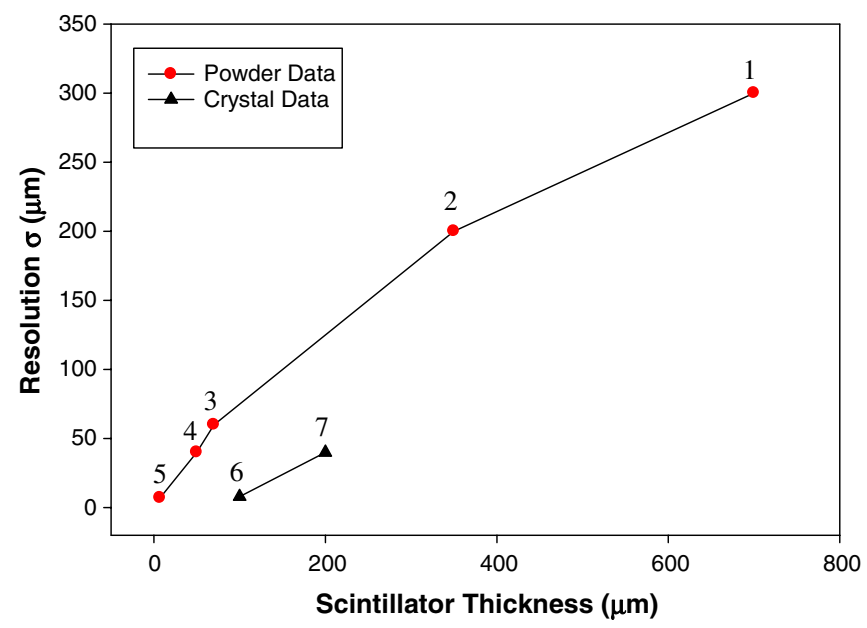

FIG. 2. Combined plot of powder and crystal screen thicknesses and deduced spatial resolution terms based on a quadrature analysis of the reported observed image sizes with the OTR or reference image size. The data points with relativistic electron beams are collected here: (1) $\mathrm{Al}_{2} \mathrm{O}_{3}: \mathrm{Cr}$ at Elettra [15]; (2) $\mathrm{Al}_{2} \mathrm{O}_{3}: \mathrm{Cr}$ at ANL [14]; (3) YAG:Ce at Fermilab (this paper); (4) YAG:Tb at BNL [19]; and (5) our estimate for a screen at the 5 - $\mu \mathrm{m}$ grain size at 45 degrees. The scintillator crystal points are from (6) YAG:Ce at SCSS [10] and (7) YAG:Ce at Mainz [25], in both latter cases the crystal surface plane was at 90 degrees to the beam direction while points 1,2 , and 3 were at 45 degrees. The lines between points are used to guide the eye. differences in observed beam image sizes by postulating there was a powder screen term that could be treated in quadrature with the actual beam size as in Eq. (1). As shown in Fig. 2, there is a strong indication of the thickness effect in these merged examples of data from four different labs. Our present YAG:Ce result using the screen thickness at 45 degrees (effectively $70 \mu \mathrm{m}$ thick) is in basic agreement with the deduced resolution result of $40 \mu \mathrm{m}$ at $60 \mathrm{MeV}$ with $500 \mathrm{pC}$ charge [19] and for an assumed similarly deposited YAG:Tb screen, albeit used with its surface normal to the beam direction with light collected at the back angle via an annular metallic lens. More comprehensive studies of YAG:Ce ceramic screens' yield, resolution, thickness effects, etc. have been done in the x-ray imaging community [20], but the relativistic electron beams are more penetrating than the $100-\mathrm{keV}$ x rays typically used so we cannot directly apply the x-ray results in all parameters. As stated earlier, we have now changed to the single crystal screens with sub-10- $\mu \mathrm{m}$ resolution that basically do not have grain size or light scattering issues of the same type as that of the powder versions of YAG:Ce.

\section{B. Camera resolution and depth-of-focus effects}

In the case of the divergence measurements based on the slit sampling, we have determined that the depth of focus of the optics and the 45-degree screen orientation was an issue. If one samples the phase space with 1-mm slit spacing, the outer slit images are blurred by the degraded system resolution. This effect is graphically illustrated in Fig. 3, where we see that the observed slit-image size variation across the field of view can be reproduced by adding the $z$-dependent optical-bench-based cameraresolution term in quadrature with the minimum slit image seen at the focus for the $\mathrm{X} 5$ case. We see that from the central in-focus reference position for X5, even the next slit-image position has a detectable growth. An even larger

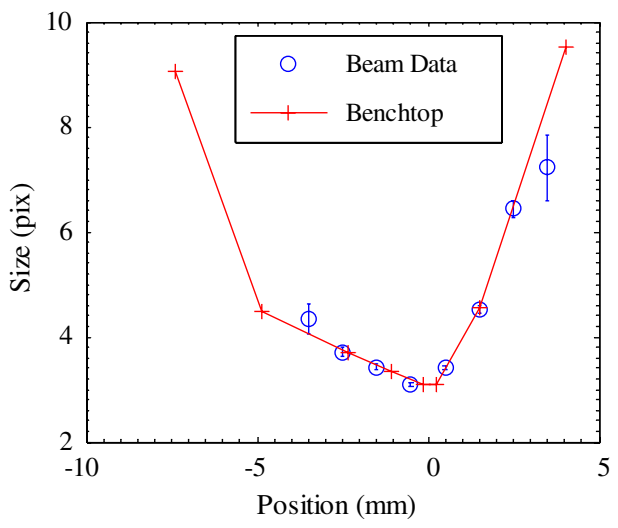

FIG. 3. Comparison of the observed vertical slit-image sizes and calculated sizes from the bench tests depth-of-focus data added in quadrature with the minimum beam size at focus for X5. 
effect was seen at X24 where the slit-image spacings were $3 \mathrm{~mm}$. To address such depth-of-focus effects in A0PI stations, we have installed at X5 and X24 a YAG:Ce single crystal with its surface plane normal to the beam direction and with a 45-degree mirror just downstream which directs the light to the optics. The resulting slit images were more uniform in size across the scene, and averaged divergences (and thus calculated emittances) were lower in subsequent tests.

\section{EXPERIMENTAL RESULTS}

Following our implementation of the diagnostic station upgrades, we returned to the basic EEX experiments. Example beam images are shown in Figs. 4(a) and 4(b) for screens X3 and X5. The beam image and vertical slitimage profiles were fit to Gaussian shapes as shown in Figs. 4(c) and 4(d). Typical full image sizes were 1-2 mm, but the slit images can be as small as $80 \mu \mathrm{m}$ in size. We did not observe any unusual, spatially localized enhancements in the beam images that could be attributed to the microbunching instability as reported in other linacs, but only after chicane bunch compression.

The evaluation of phase space can be visualized by plotting the calculated divergences for each slit-image sample and constructing the 2D $x-x^{\prime}$ phase space [12] as shown in Fig. 5. In Fig. 5(a) the measured divergences at each slit position are plotted, while in Fig. 5(b) the interpolation of those data points, weighted by intensity and using a Gaussian shape, is shown. In addition, from such data the Courant-Snyder parameters $(\alpha, \beta, \gamma)$ were also determined and used as input for the emittance-exchange model. Additional X3-X5 results are shown in Fig. 6. The partition of beam size (red circles) and divergence
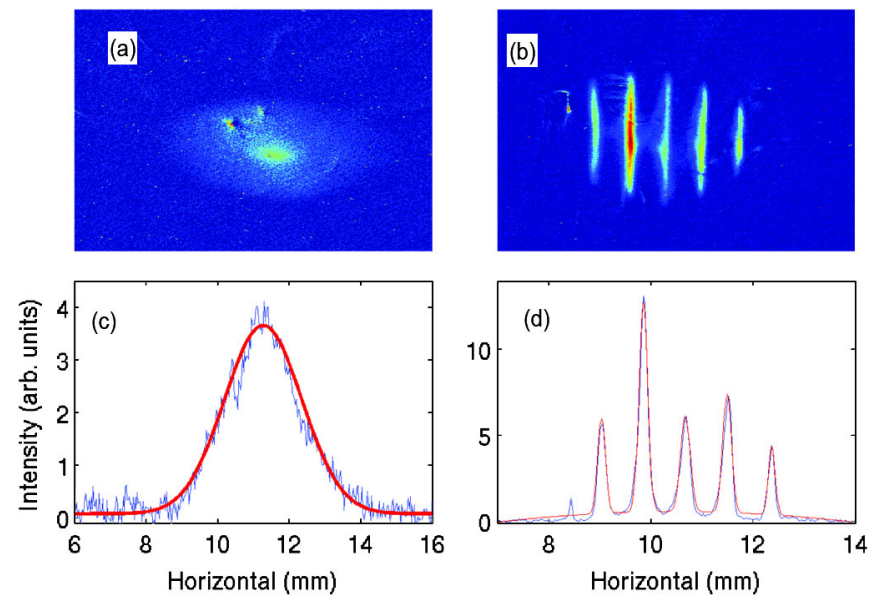

FIG. 4. Composite display of (a) X3 OTR image, (b) X5 YAG: Ce crystal slit images, (c) projected $\mathrm{x}$ profile (blue) and its Gaussian profile fit (red curve), and (d) slit-image $x$ profiles with the Gaussian fitted curves (red).
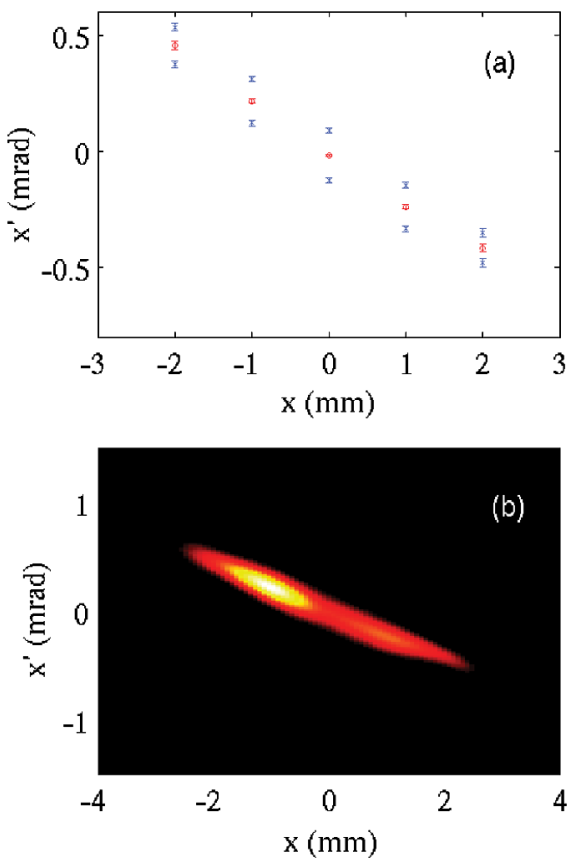

FIG. 5. Plots of (a) the measured $x$ - $x^{\prime}$ phase space and (b) visualization of transverse phase space based on the slit sampling of the beam cross section at X3 and the slit images being observed at X5 as shown in Fig. 4.

(blue triangles) varied with main solenoid (S2) current while the $x$ emittances (black circles) were calculated as $2.0-2.6 \mathrm{~mm} \mathrm{mrad}$. The lowest divergence numbers result from large fractional corrections for the camera-resolution term so we ran in the solenoidal-current regime where the errors were fractionally smaller. The upgraded X6 station with a larger drift distance from the X3 station, and hence larger divergence-related effects, will ameliorate this issue as will be separately reported [21].

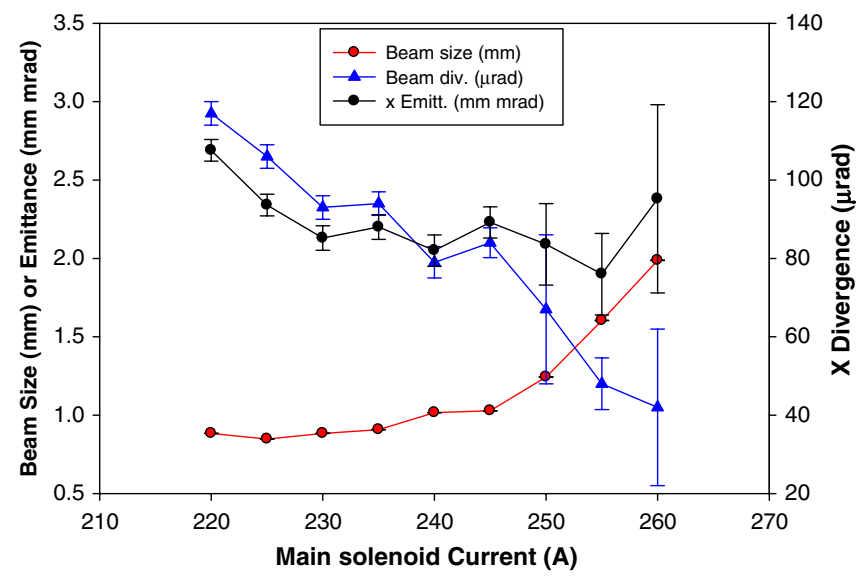

FIG. 6. The variation of beam size (red circles), divergence (blue triangles), and normalized $x$ emittances (black squares) at X3-X5 versus the main solenoid current. 


\section{EMITTANCE-EXCHANGE RESULTS}

The incoming longitudinal emittance was determined by tuning the nine-cell cavity rf phase for minimum energy spread, as observed at the XS3 screen in the straight line spectrometer. At this phase setting, the bunch length was determined at the X09 OTR screen using a Hamamatsu C5680 streak camera operating with synchroscan vertical plug-in unit that was phase locked to $81.25 \mathrm{MHz}$ [22]. The standard input optics barrel using transmissive fused silica lenses was replaced with a reflective mirror optics configuration to reduce the contributions of chromatic temporal dispersion to the observed bunch lengths. Typical energy spread and bunch-length 1-sigma values are $8-10 \mathrm{keV}$ and 3 ps or $0.9 \mathrm{~mm}$, respectively. Under the assumption of an upright longitudinal phase space, the product of these rms values would give longitudinal emittance.

For the outgoing transverse emittances, the beam size was first determined at X23, and then with the slit assemblies inserted at X23, the slit images were measured at $\mathrm{X} 24$. The X23-X24 image pairs were used with a drift of $0.56 \mathrm{~m}$. The outgoing emittances and C-S parameters were calculated with the same MATLAB program used for the input emittances [12].

The outgoing longitudinal emittance was evaluated by an energy-spread measurement at the XS4 screen in the vertical spectrometer and by the bunch-length measurement at X24 with the OTR transported to the same streak camera as used at X09 and/or with the X24 far-infrared coherent transition radiation transported to a MartinPuplett interferometer [23]. After the exchange, bunch lengths are typically sub-ps and the energy spread is about 5-8 keV. Because of optics constraints and the limitations of the longitudinal measurement (i.e., no deflecting cavity), it was difficult to establish without ambiguity the required upright phase-ellipse conditions so we only have an upper limit formed by the product of the projected components. Examples of the OTR streak image and bunch-length profile obtained at X24 with the five-cell rf cavity off (left) and on (right) are shown in Fig. 7. The initial bunch-length sigma was $2.1 \mathrm{ps}$ and the EEX one was $\sim 0.7$ ps under these conditions. Figure 8 shows the tracking of 25 shots with the five-cell cavity off (red dots) and five-cell cavity on (blue squares). The results are quite consistent, but the scatter of points is larger in the cavityon data since the corrections to the raw data are fractionally larger than for the cavity-off data. The intrinsic camera resolution is $0.6 \mathrm{ps}(\sigma)$ for a red laser source. Although the system resolution with OTR is larger, we are still able to apply the bandwidth correction with mirror optics to detect e-beam bunch lengths at the 0.6 ps level $[22,23]$.

As further evidence for the EEX process, we show the observed energy-spread results at XS3 (before) and at XS4 (after EEX) in Fig. 9. In this case the spectrometer focal plane screens had also been changed to the YAG:Ce single crystal with its surface normal to the beam direction
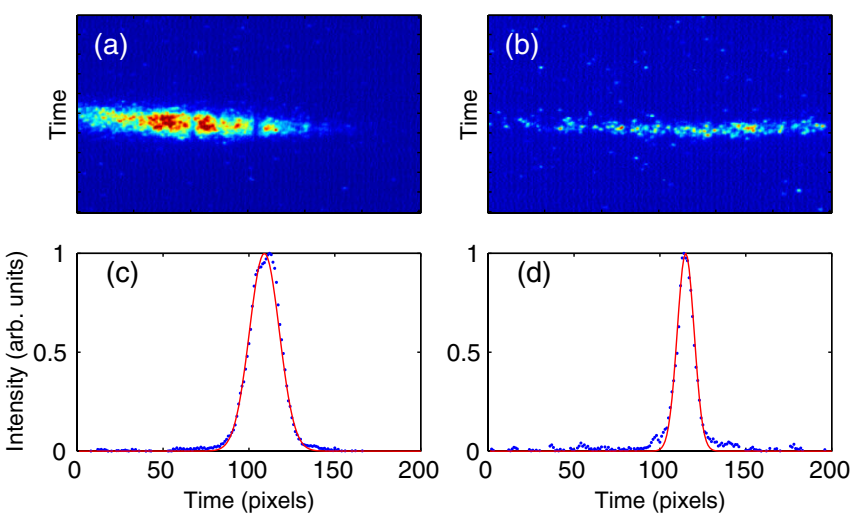

FIG. 7. Initial comparison of the OTR streak images taken at X24 (a) with the five-cell cavity power off and (b) with cavity power on for EEX. The projected temporal profiles (blue dots) are shown below the images in (c) and (d), respectively. The red curve is the Gaussian fit to the data profile in each.

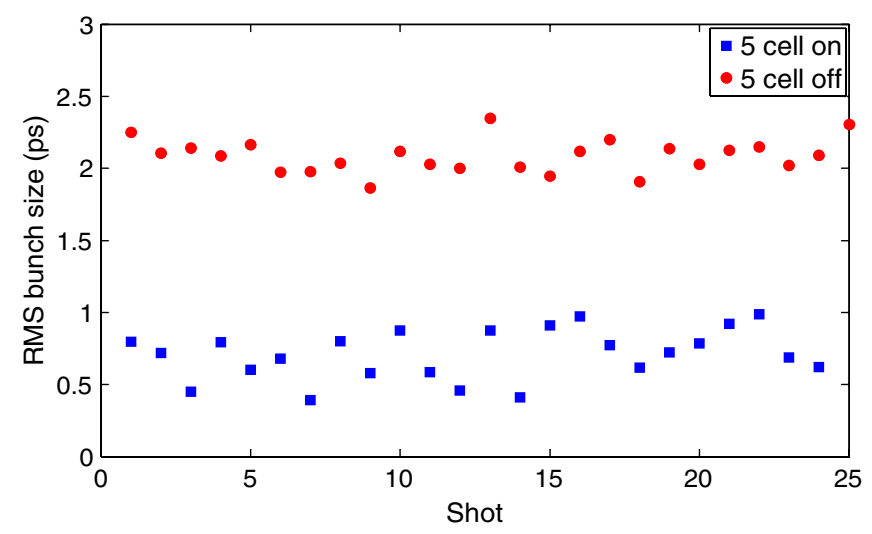

FIG. 8. Comparison of the streak camera results at X24 for the five-cell cavity off (red dots) and on (blue squares) when the exchange was tuned for minimum delta $t$.

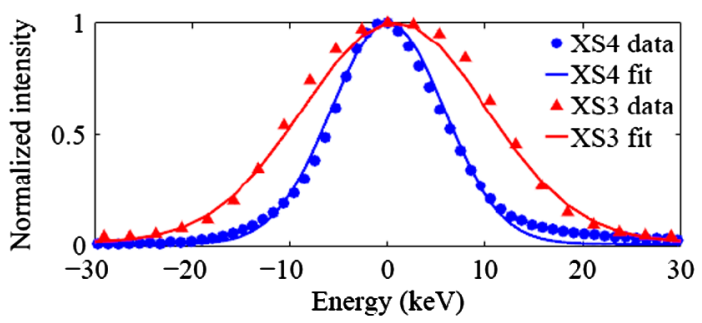

FIG. 9. Energy-spread measurements before and after EEX with the upgraded screens. The triangles show typical incoming minimum energy spread as measured at XS3 with a Gaussian fit to the projection. After EEX, the energy spread measured at XS4 is shown with dots and a Gaussian fit to the projection.

followed by the downstream $45^{\circ}$ mirror. The screen resolution term and depth-of-focus aspects thus were reduced to negligible compared to the camera-resolution term. The observed XS4 energy spread of $6.1 \pm 0.6 \mathrm{keV}$ is clearly 
TABLE III. Summary of EEX results at $14.3 \mathrm{MeV}$ and $250 \mathrm{pC}$ charge per bunch using the upgraded transverse profile diagnostics and identified corrections.

\begin{tabular}{lcc}
\hline \hline Normalized emittance (mm mrad) & Initial & Outgoing \\
\hline$\varepsilon_{x}$ & $2.6 \pm 0.3$ & $11 \pm 2$ \\
$\varepsilon_{z}$ & $11.5 \pm 1.5$ & $3.1 \pm 0.3$ \\
$\varepsilon_{y}$ & $2.5 \pm 0.3$ & $3.7 \pm 0.4$ \\
\hline \hline
\end{tabular}

smaller than the initial 9.2 $\pm 0.9-\mathrm{keV}$ value, and the direct comparison of the projected profiles confirms this. Thus, both longitudinal phase-space components are clearly reduced in value by the exchanger beam line when the five-cell cavity is properly powered at the $100 \%$ gradient. These EEX data in Figs. 8 and 9 cannot be explained by a scenario of a simple phase-space rotation that could reduce one or the other of the projected values. In addition, the same exchanger beam line has also been used by our team to transform a horizontal intensity modulation into a longitudinal modulation or sub-ps pulse train as reported previously [24]. This latter demonstration further validates our EEX beam line setup procedures.

We proceeded to test emittance exchange, and took advantage of the newly installed YAG:Ce crystals at X5 and X24 to measure the transverse parameters. The results are shown in Table III with transverse $x$ and longitudinal emittances shown in adjacent rows to facilitate comparison of the exchanged emittances and with the $y$ emittances shown in the last row. The initial values and outgoing values are tabulated.

The exchange of the transverse $(x)$ and longitudinal emittances are clear, and they are close to one to one within the errors in both exchange directions. The outgoing longitudinal-emittance value was evaluated at minimum energy spread observed in XS4 with screen corrections applied. In this case the outgoing bunch length is $\sim 25 \%$ larger than in Fig. 8, but the longitudinal-emittance product is smaller than when using the minimum bunch-length settings. Subsequent tests with the crystal screen in XS4 support this value [21]. The vertical emittance growth was about $20 \%$ even with the 1-sigma errors applied, perhaps due to some coupling between the $x-y$ planes. The statistical errors are calculated in the transverse emittance code [12], and the longitudinal errors are based on the statistical variances on multiple samples of the bunch lengths and the energy spread.

\section{SUMMARY}

In summary, we have investigated the contributions of several terms to beam profile station system resolution at A0PI and converted most of the YAG:Ce powder screens to YAG:Ce single crystal screens. We have also changed the geometry of the screens at the divergence stations and, as of recently, the spectrometer stations to eliminate the compromising depth-of-focus issues across the field of view. The bunch-length diagnostics had been previously upgraded [22,23]. These critical diagnostic upgrades have resulted in more reliable characterization of the transverse and longitudinal emittances before and after EEX (with an almost factor of 2 reduction in the emittance values compared to the preliminary report of Ref. [7] in which no projected profile size corrections were made and no diagnostics upgrades had been done). Subsequently, more recent measurements led to the long-sought observations of nearly ideal one-to-one exchanges with little evidence of coupling into the $y$ plane [21].

\section{ACKNOWLEDGMENTS}

The authors acknowledge the beam line vacuum work of W. Muranyi and B. Tennis and support from M. Wendt, N. Eddy, M. Church, and E. Harms of Fermilab. They also acknowledge K. Floettmann of DESY for providing the YAG:Ce powder screens and P. Piot of NIU and F. Stephan of PITZ for discussions on their application. This work was supported by U.S. Department of Energy, Office of Science, Office of High Energy Physics, under Contract No. DE-AC02-06CH11357.

[1] M. Cornacchia and P. Emma, Phys. Rev. ST Accel. Beams 5, 084001 (2002)

[2] P. Emma, Z. Huang, and K.-J. Kim, Phys. Rev. ST Accel. Beams 9, 100702 (2006).

[3] J. P. Carneiro et al., Phys. Rev. ST Accel. Beams 8, 040101 (2005).

[4] T.W. Koeth, Ph.D. thesis, Rutgers University, 2009.

[5] L. Wartski et al., J. Appl. Phys. 46, 3644 (1975).

[6] R. B. Fiorito and D.W. Rule, AIP Conf. Proc. 319, 21 (1994).

[7] T. W. Koeth et al., in Proceedings of the 23rd Particle Accelerator Conference, Vancouver, Canada, 2009 (IEEE, Piscataway, NJ, 2009), TU4PBI01.

[8] A.H. Lumpkin et al., in Proceedings of the 31st International Free Electron Laser Conference (FEL 09), Liverpool, UK (STFC Daresbury Laboratory, Warrington, 2009), TUPC46.

[9] B.X. Yang (private communication).

[10] M. Maesaka et al., in Proceedings of the 10th European Workshop on Beam Diagnostics and Instrumentation for Particle Accelerators, Basel Switzerland (DIPAC09) (2009), MOOA03.

[11] C. H. Wang et al., International Conference on Accelerator and Large Experimental Physics Control Systems, Trieste Italy (ICALEPS) (1999), p. 284.

[12] R. Thurman-Keup, A. S. Johnson, A. H. Lumpkin, and J. Ruan, in Proceedings of the 2011 Particle Accelerator Conference, New York, NY (PAC11) (to be published).

[13] L. Lyons, Statistics for Nuclear and Particle Physicists (Cambridge University Press, Cambridge, 1986). 
[14] A. H. Lumpkin, W. J. Berg, B. X. Yang, and M. White, in Proceedings of LINAC 98 (1999), TU4049.

[15] Silvano Bassanese et al., in Proceedings of the 11th European Particle Accelerator Conference, Genoa, 2008 (EPS-AG, Genoa, Italy, 2008), TUPC004.

[16] Klaus Floettmann (private communication). He has provided such Ce-doped and Tb-doped YAG powder screens to BNL and FNAL over this past decade.

[17] M. Castellano and V. A. Verzilov, Phys. Rev. ST Accel. Beams 1, 062801 (1998).

[18] A.H. Lumpkin et al., Proceedings of the 2010 Beam Instrumentation Workshop, Santa Fe NM (BIW10) (2010), TUPSM001.

[19] A. Murokh, J. Rosenzweig, I. Ben-Zvi, X. Wang, and V. Yakimenko, in Proceedings of the 19th Particle Accelerator Conference, Chicago, Illinois, 2001 (IEEE, Piscataway, NJ, 2001), p. 1333.
[20] I. Kandarakis et al., Nucl. Instrum. Methods Phys. Res., Sect. A 538, 615 (2005).

[21] J. Ruan, A. S. Johnson, A. H. Lumpkin, R. Thurman-Keup, H. Edwards, R. P. Fliller, T. W. Koeth, and Y. E. Sun, Phys. Rev. Lett. (to be published).

[22] A. H. Lumpkin and J. Ruan, Proceedings of the 2008 Beam Instrumentation Workshop, Lake Tahoe CA (BIW08) (2009), TUPTPF062.

[23] R. M. Thurman-Keup et al., Proceedings of the 2008 Beam Instrumentation Workshop, Lake Tahoe CA (BIW08) (2009), TUPTPF025.

[24] Y.-E. Sun, P. Piot, A. Johnson, A. H. Lumpkin, T. J. Maxwell, J. Ruan, and R. Thurman-Keup, Phys. Rev. Lett. 105, 234801 (2010).

[25] G. Kube, C. Behrens, and W. Lauth, Proceedings of 2010 International Particle Accelerator Conference (IPAC2010) (2010), MOPD088, p. 906. 